\title{
A Numerical Investigation on Cumulative Sum of the Liouville Function
}

\author{
Minoru TANAKA \\ Gakushuin University
}

Let $\lambda(n)$ be the Liouville function defined as $\lambda(n)=(-1)^{\nu}$, where $\nu$ is the number of prime factors of a positive integer $n$, multiple factors being counted according to their multiplicity. Thus $\lambda(1)=1, \lambda(2)=-1$, $\lambda(3)=-1, \lambda(4)=1, \lambda(5)=-1, \lambda(6)=1, \lambda(7)=-1, \lambda(8)=-1, \lambda(9)=1, \lambda(10)=$ $1, \cdots$.

We put

$$
L(x)=\sum_{n=1}^{x} \lambda(n)
$$

In this paper we assume $x$ to be a positive integer. Thus $L(1)=1, L(2)=$ $0, L(3)=-1, L(4)=0, L(5)=-1, L(6)=0, L(7)=-1, L(8)=-2, L(9)=-1$, $L(10)=0, \cdots$.

The object of this note is to report some numerical results obtained by the author on $L(x), x \leqq 10^{9}$, especially on how $L(x)$ changes its sign as $x$ increases from 1 to $10^{9}$.

For convenience we divide the integers $1-10^{\circ}$ into subregions each consisting of 10000 consecutive integers.

$1-10000$. In this region, $L(x)=0$ only for $x=2,4,6,10,16,26,40,96$, $586 ; L(x)>0$ only for $x=1$.

10001-906150000. Always $L(x)<0$.

906150001-906160000. $L(x)=0$ for 54 values of $x$, the first of which is $906150256 ; L(x)>0$ for 1529 values of $x$, the first of which is 906150257 .

906160001-906180000. Always $L(x)<0$

906180001-906190000. $L(x)=0$ for 16 values of $x ; L(x)>0$ for 9612 values of $x$.

906190001-906200000. $L(x)=0$ for 75 values of $x ; L(x)>0$ for 7784 values of $x$.

$906200001-906210000 . \quad L(x)=0$ for 22 values of $x ; L(x)>0$ for 9643 values of $x$. 
906210001-906470000. Always $L(x)>0$.

906470001-906480000. $L(x)=0$ for 15 values of $x ; L(x)>0$ for 9882 values of $x$.

906480001-906490000. $L(x)=0$ for 61 values of $x$; the last of which is $906488080 ; L(x)>0$ for 6976 values of $x$; the last of which is 906488079 .

Thus $L(x)<0$ from $x=587$ to $x=906150255$, and from $x=906488081$ to $x=10^{\circ}$.

$L(x)$ takes sometimes negative values, and sometimes equals zero from $x=2$ to $x=586$. $L(x)$ takes sometimes positive, sometimes negative values, and sometimes equals zero from $x=906150256$ to $x=906488080$.

This time we divide the integers $1-10^{\circ}$ into subregions each consisting of $10^{8}$ consecutive integers. We give a short list of minimum and maximum values of $L(x)$ for each of the subregions.

$$
\begin{array}{llll}
\min =-10443 & \max =1 & \left(x \leqq 10^{8}\right) & L\left(10^{8}\right)=-3884 \\
\min =-17847 & \max =-1497 & \left(10^{8}<x \leqq 2 \cdot 10^{8}\right) & L\left(2 \cdot 10^{8}\right)=-11126 \\
\min =-19647 & \max =-2262 & \left(2 \cdot 10^{8}<x \leqq 3 \cdot 10^{8}\right) & L\left(3 \cdot 10^{8}\right)=-16648 \\
\min =-19496 & \max =-2016 & \left(3 \cdot 10^{8}<x \leqq 4 \cdot 10^{8}\right) & L\left(4 \cdot 10^{8}\right)=-11200 \\
\min =-28531 & \max =-9240 & \left(4 \cdot 10^{8}<x \leqq 5 \cdot 10^{8}\right) & L\left(5 \cdot 10^{8}\right)=-18804 \\
\min =-19836 & \max =-2842 & \left(5 \cdot 10^{8}<x \leqq 6 \cdot 10^{8}\right) & L\left(6 \cdot 10^{8}\right)=-15350 \\
\min =-28731 & \max =-12250 & \left(6 \cdot 10^{8}<x \leqq 7 \cdot 10^{8}\right) & L\left(7 \cdot 10^{8}\right)=-25384 \\
\min =-29736 & \max =-12701 & \left(7 \cdot 10^{8}<x \leqq 8 \cdot 10^{8}\right) & L\left(8 \cdot 10^{8}\right)=-19292 \\
\min =-20870 & \max =-3158 & \left(8 \cdot 10^{8}<x \leqq 9 \cdot 10^{8}\right) & L\left(9 \cdot 10^{8}\right)=-4630 \\
\min =-27756 & \max =829 & \left(9 \cdot 10^{8}<x \leqq 10^{9}\right) & L\left(10^{8}\right)=-25216
\end{array}
$$

Comments on literature. Lehman [2] found that $L(906180359)=1$, but he could not ascertain whether $x=906180359$ is the integral value of $x$ next to 1 for which $L(x)>0$ or not.

Comments on theoretical results. Tanaka [3] contains a proof that $L(x)-B \sqrt{x}$ changes its sign infinitely often as $x$ tends to infinity, where $B=1 / \zeta(1 / 2)=-0.68477$. But it is an open problem until the present whether $L(x)$ itself changes its sign infinitely often or not. In connection with this problem, see Ingham [1].

The author would like to thank the staff of the Computer Center of Gakushuin University for their assistance given to him during the work for the paper. 


\section{References}

[1] A. E. INGHaM, On two conjectures in the theory of numbers, Amer. J. Math., 64 (1942), 313-319.

[2] R. S. Lemman, On Liouville's function, Math. Comp., 14 (1960), 311-320.

[3] M. TANakA, On the Möbius and allied functions, Tokyo J. Math., to appear.

Present Addres8:

Department of Mathematics

GAKUSHUIN UNIVERSITY

MeJiro, Toshima-KU

TOKYO 171 